\title{
Esophageal Metastasis from Rectal Cancer Successfully Treated with Fluorouracil-Based Chemotherapy with Bevacizumab: A Case Report and Review of the Literature
}

\author{
Sho Watanabe Atsuo Takashima $^{a}$ Hirokazu Taniguchi ${ }^{b}$ \\ Yusaku Tanaka ${ }^{c}$ Shoko Nakamura ${ }^{a}$ Natsuko Okita ${ }^{a}$ Yoshitaka Honma ${ }^{a}$

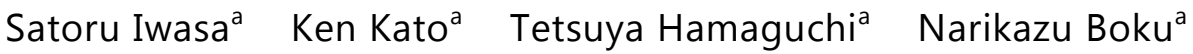 \\ ${ }^{a}$ Department of Gastrointestinal Medical Oncology, National Cancer Center Hospital, \\ Tokyo, Japan; ${ }^{b}$ Department of Pathology and Clinical Laboratories, National Cancer \\ Center Hospital, Tokyo, Japan; ' Department of Endoscopy, National Cancer Center \\ Hospital, Tokyo, Japan
}

\section{Keywords}

Colorectal cancer · Esophageal metastasis · Esophageal cancer · Endoscopy · FOLFOX ·

FOLFIRI $\cdot$ Bevacizumab

\begin{abstract}
Esophageal metastasis from colorectal carcinoma is uncommon, and diagnosis of esophageal metastasis is difficult. We report a case of a 54-year-old woman with postoperative recurrence of rectal cancer metastasizing to the esophagus. She underwent rectectomy and adjuvant chemotherapy with fluorouracil, leucovorin plus oxaliplatin for stage IIIB rectal cancer. Three years later, she presented with dysphagia and cough. Computed tomography showed thickening of the esophagus wall, enlargement of the lymph nodes in the mediastinum and
\end{abstract}




\section{Case Reports in Oncology}

abdomen, and ground-glass opacities in the right lung. Endoscopy revealed a submucosal tumor of the midthoracic esophagus. Histopathological analysis of the tumor biopsy showed infiltration of adenocarcinoma cells into the stroma of the esophagus; tumor cells were positive for caudal type homeobox 2 and negative for thyroid transcription factor 1 . A transbronchial biopsy indicated pulmonary lymphangitic carcinomatosis of rectal adenocarcinoma. Based on those findings, she was diagnosed with recurrent rectal cancer. She received fluorouracil-based chemotherapy plus bevacizumab, which ameliorated her symptoms and induced a durable response without severe adverse events. Diagnosis of esophageal metastasis from rectal cancer can thus be made by repeated biopsy. Furthermore, aggressive systemic treatment with fluorouracil-containing chemotherapy and bevacizumab is a treatment option for colorectal cancer patients with esophageal metastasis.

\section{Introduction}

Esophageal metastases of colorectal cancer are uncommon [1]. Diagnosis of metastatic esophageal carcinoma is still difficult, due to its rarity and the submucosal involvement of metastatic lesions in the esophagus. Lymphatic or vascular spread of tumor cells has been proposed to lead to the formation of metastatic esophageal tumors [2]. In patients with metastatic colorectal carcinoma, chemotherapy using targeted agents increased median survival times to more than 30 months [3]. In contrast, the outcome of patients with esophageal metastasis remains poor, despite various treatment options [4-8]. We describe a case of postoperative recurrence of rectal cancer with esophageal metastasis, in which a repeated endoscopic biopsy with immunohistochemical assessment was diagnostic, and the combination of fluorouracil-based chemotherapy and bevacizumab showed a durable tumor response and tolerability.

\section{Case Presentation}

In 2012, a 54-year-old woman had low anterior resection for rectal carcinoma followed by chemotherapy with fluorouracil/folinic acid plus oxaliplatin (FOLFOX). Preoperative laboratory tests showed normal levels of carcinoembryonic antigen (CEA) and carbohydrate antigen (CA) 19-9. Pathological examination revealed a moderately differentiated rectal adenocarcinoma with pararectal and inferior mesenteric metastatic lymph nodes (pT3N1bM0, stage IIIB, Union for International Cancer Control 7th edition). At postoperative 3 years, she presented with dysphagia and cough. A blood test showed an elevated value of CA19-9 (218 $\mathrm{U} / \mathrm{mL}$, normal range $[\mathrm{NR}] \leq 37$ ). The results of other tumor markers were negative: CEA 1.7 $\mathrm{ng} / \mathrm{mL}(\mathrm{NR} \leq 5)$, squamous cell carcinoma antigen $0.4 \mathrm{ng} / \mathrm{mL}(\mathrm{NR} \leq 1.5)$, and cytokeratin 19 fragment 3.5 ( $\mathrm{NR} \leq 3.5$ ). Computed tomography and magnetic resonance imaging of the thorax showed thickening of the esophageal wall, enlarged mediastinal and abdominal lymph nodes, and ground-glass opacities with interlobular septal thickening in the right lung, which were suggestive of primary unresectable esophageal cancer (Fig. 1a, b). Endoscopy revealed an elevated submucosal mass with white speckles on the mucosa in the middle thoracic 


\section{Case Reports in Oncology}

Case Rep Oncol 2017;10:407-415

DOI: $10.1159 / 000474939$

(C) 2017 The Author(s). Published by S. Karger AG, Basel www.karger.com/cro

Watanabe et al.: Esophageal Metastasis from Rectal Cancer Successfully Treated with Fluorouracil-Based Chemotherapy with Bevacizumab: A Case Report and Review of the Literature

esophagus (Fig. 2). Though an initial tumor biopsy performed previously at another hospital did not reveal any malignancy, she was referred to the National Cancer Center Hospital with a suspicion of esophageal cancer. A second biopsy showed adenocarcinoma beneath the normal squamous epithelium, which was similar to the pathological findings of the resected rectum. Immunohistochemically, the tumor cells were positive for cytokeratin (CK) 8, CK20, and caudal type homeobox (CDX) 2, and were negative for CK7, thyroid transcription factor 1, and p63 (Fig. 3). Based on these findings, a diagnosis of metastatic esophageal tumor from rectal carcinoma was confirmed. A transbronchial biopsy indicated pulmonary lymphangitic carcinomatosis from rectal carcinoma. Despite rapid disease progression, her oral intake and performance status were well maintained. Polymerase chain reaction of the rectum samples revealed the presence of a Kirsten rat sarcoma viral oncogene homolog codon 12 mutation, limiting the use of anti-epidermal growth factor receptor antibodies as a treatment option. Considering her good performance status, chemotherapy using FOLFOX with bevacizumab (FOLFOX/BV) was initiated as first-line chemotherapy. Four cycles of FOLFOX/BV led to rapid tumor shrinkage concomitant with symptomatic relief (Fig. 1c). After 13 cycles, she developed bone metastasis in a cervical vertebra and received palliative radiotherapy. As the esophageal lesion did not progress, she was subsequently given 4 cycles of chemotherapy with fluorouracil/folinic acid, irinotecan, and bevacizumab (FOLFIRI/BV), which induced stable disease. However, the tumor progressed, and she died 16 months after the postoperative recurrence.

\section{Discussion}

Distant metastasis of any primary cancer to the esophagus is uncommon. Autopsy studies have shown that 3.1-6.1\% of patients dying from any cancer type exhibited esophageal metastasis, with breast and lung cancer representing the most common primary sites $[9,10]$. To date, secondary involvement of the esophagus by colorectal cancer has been reported in five cases [4-8] (Table 1). Most of those patients underwent initial surgery and relapsed with new metastatic lesions in the esophagus, suggesting a unique clinical feature of the esophageal metastasis from colorectal cancer. A case series study of patients with esophageal metastasis showed a similarity between radiographic and endoscopic findings. Computed tomography images demonstrated marked esophageal wall thickening without adjacent extra-esophageal masses, and endoscopy revealed tight and smooth strictures with normal mucosa. These findings indicated that most esophageal metastatic lesions occur as submucosal tumors [2], which would be consistent with the present case.

Diagnosis of metastatic esophageal tumors is still challenging, possibly because of its rarity and difficulty in diagnosis. Malignant tumors comprise $1 \%$ of submucosal lesions in the esophagus. Only 3\% of malignant submucosal tumors were diagnosed as metastases from distant primary tumors to the upper gastrointestinal wall [11]. Although pathological assessment is paramount to provide an accurate diagnosis of these rare lesions, normal esophageal squamous epithelium covering metastatic tumor cells often causes nondiagnostic results in endoscopic biopsy [2]. Our findings underscored that in patients with suspicious malignant submucosal tumors, a repeat biopsy should be performed when an initial 


\section{Case Reports in Oncology}

Watanabe et al.: Esophageal Metastasis from Rectal Cancer Successfully Treated with

Fluorouracil-Based Chemotherapy with Bevacizumab: A Case Report and Review of the

Literature

biopsy fails to demonstrate malignant cells. Immunohistochemical staining of tumor cells in the biopsied samples is helpful in determining the primary site.

Mechanisms underlying metastasis to the esophageal wall are difficult to specify. As one of the modes of esophageal metastasis, vascular or lymphatic spread from distant primary sites has been proposed [2]. Hematogenous spread of cecal carcinoma cells has been noted in a case of esophageal metastasis, showing lack of mediastinal lymph node involvement [6]. It has also been suggested that an abundant lymphocapillary network in the submucosal layer of the esophagus enabled tumor cells from the mediastinal lymph nodes to penetrate the esophagus wall vertically or transversely, which resulted in the lymphatic spread of primary tumor [12]. In our case, metastatic mediastinal lymph nodes were found at the time of diagnosis of the recurrence, as well as pulmonary lymphangitic carcinomatosis, which was suggested to come from retrograde lymphatic spread from mediastinal lymph nodes [13]. In addition, lymphatic involvement was shown in the specimens of the resected rectum in this patient. Taken together, we hypothesize that the lymphatic spread of tumor cells via the mediastinal lymph nodes was the most likely process of esophageal metastasis in our case. Given the tendency of certain tumor types to metastasize to the esophagus, understanding the pathogenesis of metastasis on a cellular and molecular level is also required to elucidate the distinct metastatic mechanism [14].

Treatment options for colorectal cancer patients with esophageal metastasis include endoscopic stent, bypass surgery [6], chemotherapy [6-8], and palliation [5]; however, unfavorable outcomes are common due to the extensive progression of the primary cancer. In the present case, rapid tumor progress with pulmonary lymphangitic carcinomatosis necessitated prompt initiation of systemic chemotherapy for the recurrence. Bevacizumab in combination with fluorouracil-based chemotherapy (FOLFOX/BV and FOLFIRI/BV), which is a standard of care for metastatic colorectal cancer [3], resulted in a rapid and durable tumor response and symptomatic relief without severe toxicity, thus representing a treatment option for colorectal cancer patients with esophageal metastasis.

We report an uncommon case of esophageal metastasis from rectal cancer, in which repeated endoscopic biopsy with immunohistochemical assessment was diagnostic. Fluorouracil-based chemotherapy plus bevacizumab showed good efficacy and tolerability. Aggressive systemic treatment can prolong post-recurrent survival and should be considered as a treatment option for colorectal cancer patients with metastasis to the esophagus.

\section{Statement of Ethics}

All procedures followed were in accordance with the ethical standards of the ethical committee of the National Cancer Center Hospital on human experimentation and with the Helsinki Declaration of 1964 and later versions. Informed consent was obtained from the patient for being included in the study. Additional informed consent was obtained from the patient for whom identifying information is included in this article. 


\section{Case Reports in Oncology}

Case Rep Oncol 2017;10:407-415

DOI: $10.1159 / 000474939$

(C) 2017 The Author(s). Published by S. Karger AG, Basel www.karger.com/cro

Watanabe et al.: Esophageal Metastasis from Rectal Cancer Successfully Treated with Fluorouracil-Based Chemotherapy with Bevacizumab: A Case Report and Review of the Literature

\section{Disclosure Statement}

All authors declare that they have no conflict of interest. There are no funding sources to be declared.

\section{References}

1 Riihimaki M, Hemminki A, Sundquist J, Hemminki K: Patterns of metastasis in colon and rectal cancer. Sci Rep DOI: 10.1038/srep29765.

2 Simchuk EJ, Low DE: Direct esophageal metastasis from a distant primary tumor is a submucosal process: a review of six cases. Dis Esophagus 2001;14:247-250.

3 Fakih MG: Metastatic colorectal cancer: current state and future directions. J Clin Oncol 2015;33:18091824.

Fisher MS: Metastasis to the esophagus. Gastrointest Radiol 1976;1:249-251.

Lohsiriwat V, Boonnuch W, Suttinont P: Esophageal metastasis from rectal carcinoma. J Clin Gastroenterol 2005;39:744.

-6 Kagaya H, Kitayama J, Hidemura A, Kaisaki S, Ishigami H, Takei J, Kanazawa T, Nagawa H: Metastatic esophageal tumor from cecal carcinoma. Jpn J Clin Oncol 2007;37:628-631.

7 Thomasset SC, Garcea G, Berry DP: Oesophageal metastasis from colorectal cancer. Case Rep Gastroenterol 2008;2:40-44.

-8 Vashi PG, Gupta D, Tan B: Colon carcinoma with unusual metastasis to the esophagus manifesting as multiple nodules and dysphagia: management with systemic chemotherapy. Case Rep Gastroenterol 2012;6:484-488.

-9 Abrams HL, Spiro R, Goldstein N: Metastases in carcinoma; analysis of 1,000 autopsied cases. Cancer 1950;3:74-85.

10 Mizobuchi S, Tachimori Y, Kato H, Watanabe H, Nakanishi Y, Ochiai A: Metastatic esophageal tumors from distant primary lesions: report of three esophagectomies and study of 1,835 autopsy cases. Jpn J Clin Oncol 1997;27:410-414.

11 Polkowski M: Endoscopic ultrasound and endoscopic ultrasound-guided fine-needle biopsy for the diagnosis of malignant submucosal tumors. Endoscopy 2005;37:635-645.

12 Ji X, Cai J, Chen Y, Chen L-Q: Lymphatic spreading and lymphadenectomy for esophageal carcinoma. World J Gastrointest Surg 2016;8:90-94.

14 Fidler IJ: The pathogenesis of cancer metastasis: the "seed and soil" hypothesis revisited. Nat Rev Cancer 2003;3:453-458. 


\section{Case Reports in Oncology}
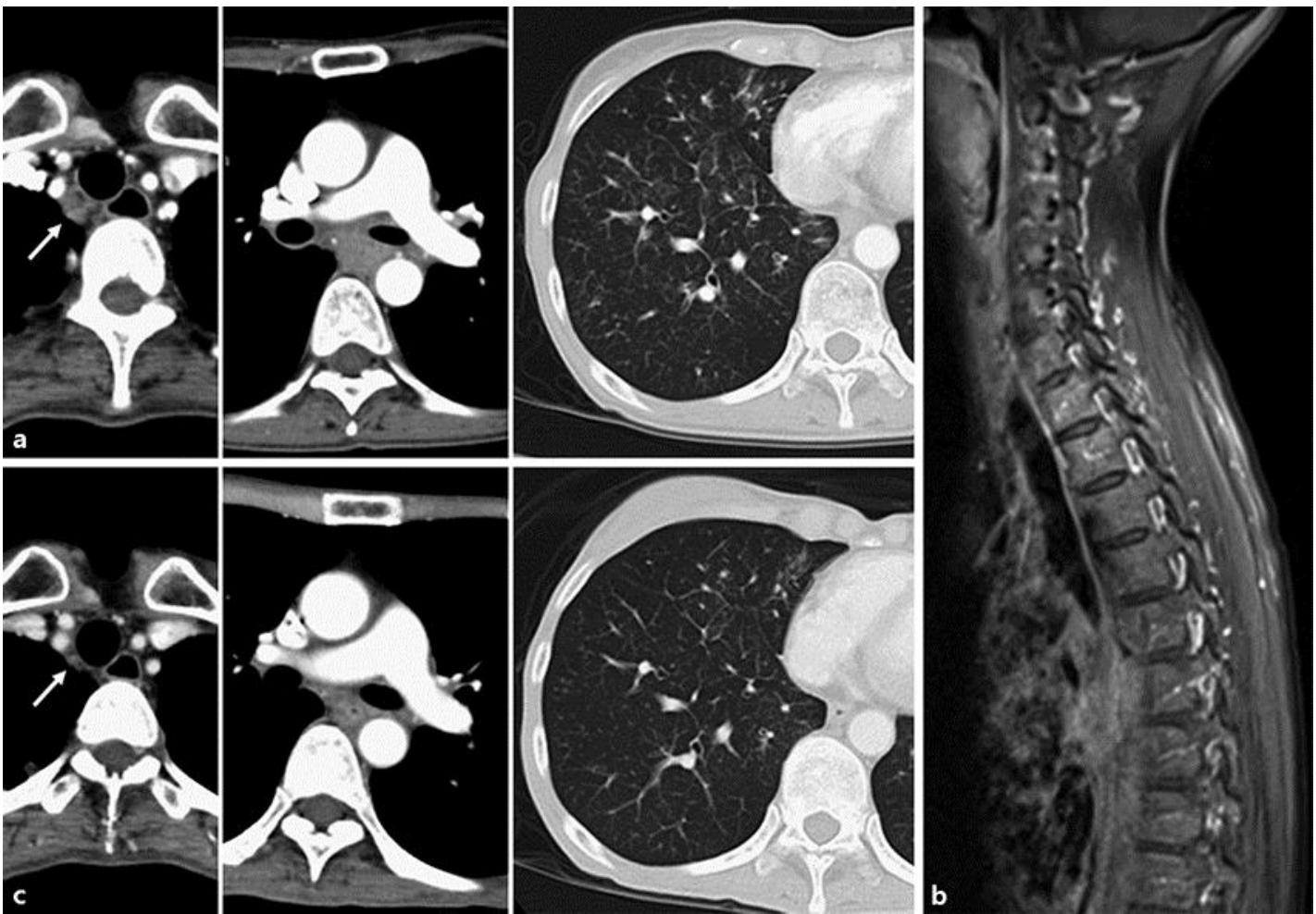

Fig. 1. Imaging features of the present case. Computed tomography of the thorax on admission (a). Mediastinal lymph node metastasis (left, arrow), thickening of the esophagus wall (middle), and ground-glass opacities with interlobular septal thickening in the right lung (right) can be seen. Magnetic resonance imaging of the thorax showed an esophageal stricture with gradual esophageal wall thickening (b). Computed tomography of the thorax after four cycles of chemotherapy (c). A tumor response was observed in the mediastinal lymph node (left, arrow), esophagus (middle), and right lung (right). 


\section{Case Reports in Oncology}

Watanabe et al.: Esophageal Metastasis from Rectal Cancer Successfully Treated with Fluorouracil-Based Chemotherapy with Bevacizumab: A Case Report and Review of the Literature

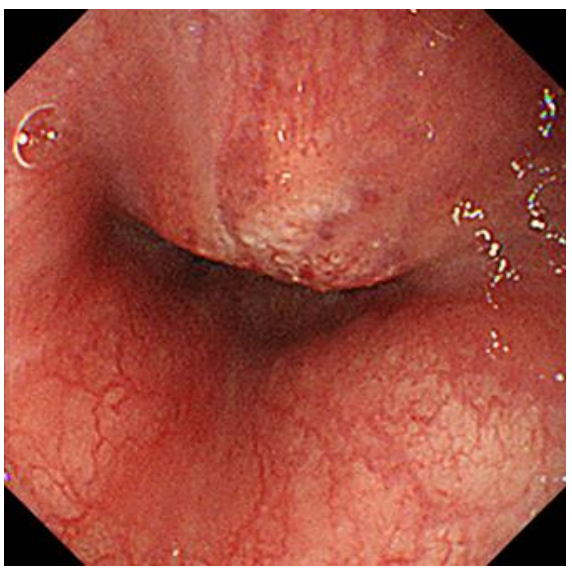

Fig. 2. Endoscopic findings. Endoscopy revealed a submucosal tumor at the middle thoracic esophagus. 


\section{Case Reports in Oncology}
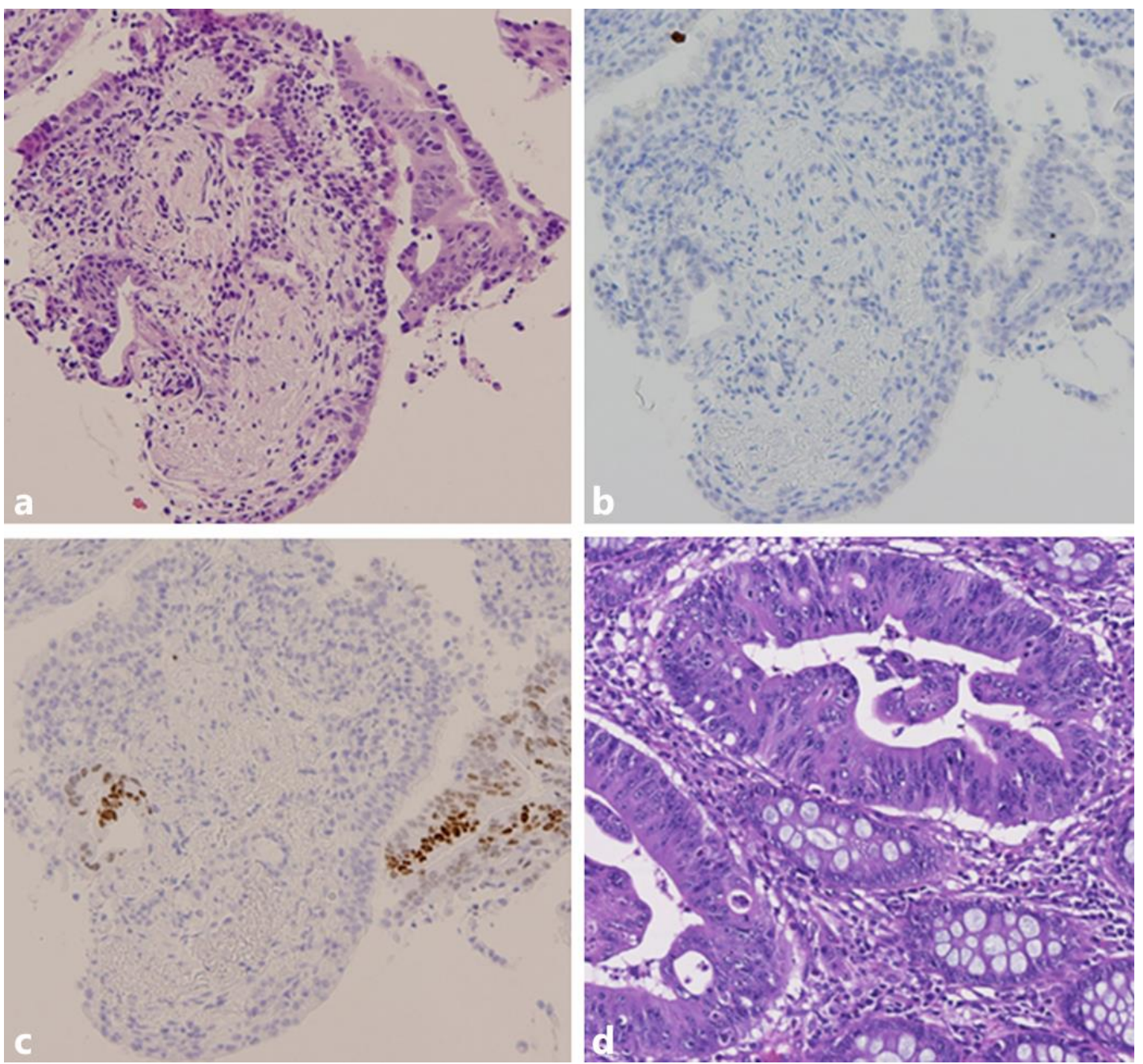

Fig. 3. Pathological findings. Biopsy samples of the esophageal tumor. Infiltrating adenocarcinoma with gland formation can be seen in the esophageal stroma (a, hematoxylin and eosin, $\times 200)$. Immunohistochemically, tumor cells were negative for thyroid transcription factor 1 (b) and positive for CDX2 (c). Moderately differentiated tubular adenocarcinoma was found in the surgical specimens of the rectum (d, hematoxylin and eosin, $\times 200$ ). 


\section{Case Reports in Oncology}

\begin{tabular}{l|l} 
DOI: $10.1159 / 000474939$ & (C) 2017 The Author(s). Published by S. Karger AG, Basel \\
\hline
\end{tabular} www.karger.com/cro

Watanabe et al.: Esophageal Metastasis from Rectal Cancer Successfully Treated with Fluorouracil-Based Chemotherapy with Bevacizumab: A Case Report and Review of the Literature

Table 1. Cases of esophagus metastasis from colorectal cancer

\begin{tabular}{|c|c|c|c|c|c|c|}
\hline $\begin{array}{l}\text { Year } \\
\text { [Ref.] }\end{array}$ & $\begin{array}{l}\text { Age, } \\
\text { years }\end{array}$ & Sex & $\begin{array}{l}\text { Primary } \\
\text { site }\end{array}$ & Initial treatment & $\begin{array}{l}\text { Treatment for } \\
\text { esophagus metastasis }\end{array}$ & $\begin{array}{l}\text { Outcome, } \\
\text { months* }\end{array}$ \\
\hline $1976[4]$ & 17 & M & Rectum & Diagnostic laparotomy & Supportive care & 2 \\
\hline $2005[5]$ & 44 & M & Rectum & $\begin{array}{l}\text { Palliative colostomy } \\
\text { 5-FU/LV }\end{array}$ & Supportive care & 2 \\
\hline 2007 [6] & 55 & M & Cecum & $\begin{array}{l}\text { Palliative colectomy } \\
5-\mathrm{FU} / \mathrm{LV}\end{array}$ & $\begin{array}{l}\text { Esophageal stent } \\
\text { Esophagectomy } \\
\text { FOLFOX }\end{array}$ & $>14$ \\
\hline 2008 [7] & 62 & M & $\mathrm{S} / \mathrm{C}$ & Sigmoid colectomy & 5-FU/LV & 6 \\
\hline 2012 [8] & 44 & M & $\mathrm{S} / \mathrm{C}$ & $\begin{array}{l}\text { Sigmoid colectomy } \\
\text { AC with FOLFOX/BV }\end{array}$ & CPT and CTX & $>3$ \\
\hline $\begin{array}{l}\text { Present } \\
\text { case }\end{array}$ & 54 & $\mathrm{~F}$ & Rectum & $\begin{array}{l}\text { Rectectomy } \\
\text { AC with FOLFOX }\end{array}$ & $\begin{array}{l}\text { FOLFOX/BV } \\
\text { FOLFIRI/BV }\end{array}$ & 16 \\
\hline
\end{tabular}

S/C, sigmoid colon; AC, adjuvant chemotherapy; 5-FU, fluorouracil; LV, leucovorin; FOLFOX, fluorouracil, leucovorin, and oxaliplatin; BV, bevacizumab; CPT, irinotecan; CTX, cetuximab; FOLFIRI, fluorouracil, leucovorin, and irinotecan. * Outcome represents survival time from detection of esophageal metastasis. 